\title{
Chorus source region localization in the Earth's outer magnetosphere using THEMIS measurements
}

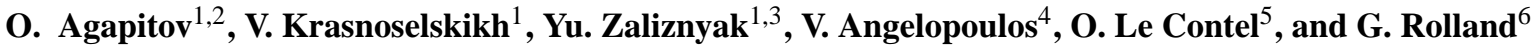 \\ ${ }^{1}$ LPCE/CNRS - University of Orleans, UMR 6115, Orleans, France \\ ${ }^{2}$ National Taras Shevchenko University of Kyiv, Kyiv, Ukraine \\ ${ }^{3}$ Institute for Nuclear Research, Kyiv, Ukraine \\ ${ }^{4}$ Institute of Geophyiscs and Planetary Physics University of California, Los Angeles (UCLA), USA \\ ${ }^{5}$ Laboratoire de Physique des Plasmas, St Maur-des-Fosses, France \\ ${ }^{6} \mathrm{CNES}$, France
}

Received: 16 February 2010 - Revised: 7 June 2010 - Accepted: 8 June 2010 - Published: 25 June 2010

\begin{abstract}
Discrete ELF/VLF chorus emissions, the most intense electromagnetic plasma waves observed in the Earth's radiation belts and outer magnetosphere, are thought to propagate roughly along magnetic field lines from a localized source region near the magnetic equator towards the magnetic poles. THEMIS project Electric Field Instrument (EFI) and Search Coil Magnetometer (SCM) measurements were used to determine the spatial scale of the chorus source localization region on the day side of the Earth's outer magnetosphere. We present simultaneous observations of the same chorus elements registered onboard several THEMIS spacecraft in 2007 when all the spacecraft were in the same orbit. Discrete chorus elements were observed at $0.15-0.25$ of the local electron gyrofrequency, which is typical for the outer magnetosphere. We evaluated the Poynting flux and wave vector distribution and obtained chorus wave packet quasiparallel propagation to the local magnetic field. Amplitude and phase correlation data analysis allowed us to estimate the characteristic spatial correlation scale transverse to the local magnetic field to be in the $2800-3200 \mathrm{~km}$ range.
\end{abstract}

Keywords. Electromagnetics (Random media and rough surfaces) - Magnetospheric physics (Plasma waves and instabilities) - Radio science (Remote sensing)

\section{Introduction}

Discrete ELF/VLF chorus emissions, the most intense electromagnetic plasma waves observed in the Earth's radiation belts and outer magnetosphere, have received increased at-

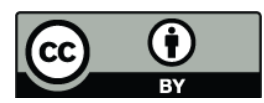

Correspondence to: O. Agapitov

(agapit@univ.kiev.ua) tention in the past several years. Characterized by rising and falling tones in the frequency range from a few hundreds to several thousands of hertz (e.g., reviews by Omura et al., 1991 and Sazhin and Hayakawa, 1992), these emissions play the significant role in local acceleration of energetic electrons in the outer radiation belts (Horne et al., 2005) and (Horne et al., 2005). Chorus is a burst-like wave emission observed in the near-Earth magnetosphere outside the plasmapause. These emissions are most often observed on the Earth's dawn side between 23:00 and 13:00 MLT (Tsurutani and Smith, 1974; Meredith, 2001, 2003). Chorus emissions, which propagate in the whistler mode, usually consist of two narrow frequency range bands centered around onehalf the electron gyrofrequency at the geomagnetic equator $\left(\omega_{\mathrm{ce}}\right)$ of the magnetic field line on which the waves are observed (Tsurutani and Smith, 1974). If present, the upper band exists in the frequency range of $\omega / \omega_{\mathrm{ce}} \approx 0.5-0.75$ and contains discrete chorus elements rising at a few $\mathrm{kHz} / \mathrm{s}$. The lower band exists in the frequency range of $\omega / \omega_{\mathrm{ce}} \approx 0.2-0.45$ and contains both elements rising at a few $\mathrm{kHz} / \mathrm{s}$ and diffuse elements.

A number of ELF/VLF chorus emissions have been observed near the magnetic equatorial plane in the dayside outer magnetosphere. Wave normal vector directions of chorus in the outer magnetosphere were determined for the first time from data obtained with the OGO5 search coil magnetometer (Burton and Holzer, 1974). In Hayakawa et al. (1990) the GEOS 1 satellite wave data have been used to determine the wave normal directions of chorus emissions at geomagnetic latitudes near $17^{\circ}$, (L-shell about 7.6). Analysis of the wave normal and Poynting vectors for each element of the emissions has shown that these emissions are generated in the geomagnetic equator and propagate to higher latitudes in a non-ducted whistler mode (Burton and Holzer, 
1974; Hayakawa et al., 1990; Yagitani et al., 1999; Inan et al., 2004). The GEOTAIL spacecraft has observed many ELF/VLF chorus emissions in the Earth's outer magnetosphere with the Plasma Wave Instrument (PWI) (Nagano et al., 1996).

In the inner and middle magnetosphere the onset of wave generation has been associated with substorm electron injections (Goldstein and Tsurutani, 1984). The source of the waves in the outer dayside region within a few $R_{\mathrm{E}}$ of the magnetopause is less well understood. In the radiation belts, chorus is believed to be generated through electron cyclotron instability by anisotropic distributions of energetic electrons in the 5 to $150 \mathrm{keV}$ range. In the outer magnetosphere simultaneously measured wave and particle data captured onboard the GEOTAIL spacecraft for chorus emissions in the dayside outer magnetosphere were analyzed in (Yagitani et al., 1999). The anisotropy observed onboard GEOTAIL was too small to generate the observed chorus emissions by the linear cyclotron resonance mechanism. The co-existence of hiss and chorus has been observed frequently by satellites in the outer magnetosphere (Hattori et al., 1991). A mechanism for hiss-triggered chorus based on GEOS-1 observations was proposed by Hattori et al. (1991).

The size of the generation region can be used to investigate and choose the generation mechanism. Before the Cluster mission, observations of ELF/VLF chorus emissions were carried out mainly by single spacecraft (Trakhtengerts, 1999). Considerable simultaneous chorus data were collected by ISEE1 and ISEE2 spacecraft (Gurnett et al., 1979). Although the correlation scale based on comparison of spectrograms from ISEE1 and ISEE2 was estimated to be in the range of units of hundred kilometers.

Recent Poynting flux and polarization measurements on board the Cluster spacecraft confirmed not only that the chorus source is located close to the equatorial plane (Santolik and Gurnett, 2003; Santolik et al., 2005; Parrot et al., 2003) but also that the dimension of the chorus source region measured along the magnetic field lines is $3000-5000 \mathrm{~km}$ (Santolik and Gurnett, 2003). The radiation belt chorus generation region scales were estimated theoretically (Helliwell, 1967; Trakhtengerts, 1999) and experimentally (Santolik and Gurnett, 2003; Breneman et al., 2009) using data from coordinated CLUSTER observations. The problem is to determine the source size given the signal distortion due to whistler wave propagation through the random irregularities of the medium. Santolik and Gurnett (2003), and Breneman et al. (2009) determined the correlation lengths of chorustype whistler waves based on multi-point Cluster WBD measurements near the chorus source region during the magnetic storm of 18 April 2002. Correlation scales were obtained from the dependence of the chorus wave amplitude correlation coefficient on the distance between Cluster spacecraft. Correlation was found to be significant throughout a range of separation distances $60-260 \mathrm{~km}$ parallel to and 7-100 km perpendicular to the background magnetic field line. At these scales, the correlation coefficient depends weakly on parallel separation and decreases with perpendicular separation. It varies between approximately 60 and $120 \mathrm{~km}$ for different data intervals. The spatial scales obtained were explained by a chorus generation region transverse to the spatial scales of the background magnetic field (Santolik et al., 2003).

In this paper we address the transverse dimensions of chorus sources using simultaneous observations of intense chorus by five THEMIS spacecraft (THA, THB, THC, THD, THE) before they were finally deployed into their designated orbits. The analysis is done for regions close to the geomagnetic equator at a radial distance of $8-9 R_{\mathrm{E}}$ which is not covered by previous work using CLUSTER data with new technique based on nonstationarity of the mutual statistic characteristics. We correlate and analyze waveform data from the five spacecraft at different separations. Our study is based mainly on high-resolution, three-axis magnetic waveform data from the search coil magnetometer (SCM) (Le Contel, 2008) and two-axis electric waveform data from the electric field wave instrument (EFW) (Bonnell et al., 2008). We also use supporting data from the electrostatic analyzer (ESA) and spin-averaged measurements from the flux-gate magnetometers (FGM) (Auster et al., 2008).

The paper is organized as follows: in section two the THEMIS SCM and EFW data description is presented and the description of processed chorus activity is proposed. In section three correlation analysis of the multi-point measurements of the whistler waveform is presented. The technique for numerical calculation of the transverse correlation scale of the electromagnetic wave refractive index fluctuation and the distance to the source region is proposed. In section four the results are summarized.

\section{Data description and processing technique}

THEMIS (Time History of Events and Macroscale Interactions during Substorms) consists of five identicallyinstrumented spacecraft, launched on 17 February 2007. The main goal of this mission is to conduct multi-point investigations of substorm phenomena in the magnetotail of the Earth's magnetosphere (Sibeck et al., 2008). During the coast phase of the mission between 17 February 2007 and 15 September 2007, all five THEMIS spacecraft were lined up in the same orbit with apogee at $15.4 R_{\mathrm{E}}$ and perigee at $1.3 R_{\mathrm{E}}$. Orbital inclination to the ecliptic plane was $13.7^{\circ}$. The THEMIS spacecraft were nearly radially aligned. During 2007 the THEMIS orbit apogees rotated through the dayside of the Earth's magnetosphere, such that in April 2007 the orbit skimmed the dusk magnetopause.

For the current study, search coil magnetometer (SCM) observations (Le Contel, 2008) and plasma measurements from the electric field instrument (EFI) (Bonnell et al., 2008) were analyzed. Waveforms in the wave burst mode (on-board trigger) with $8192 \mathrm{samp} / \mathrm{s}$ were processed. For additional 


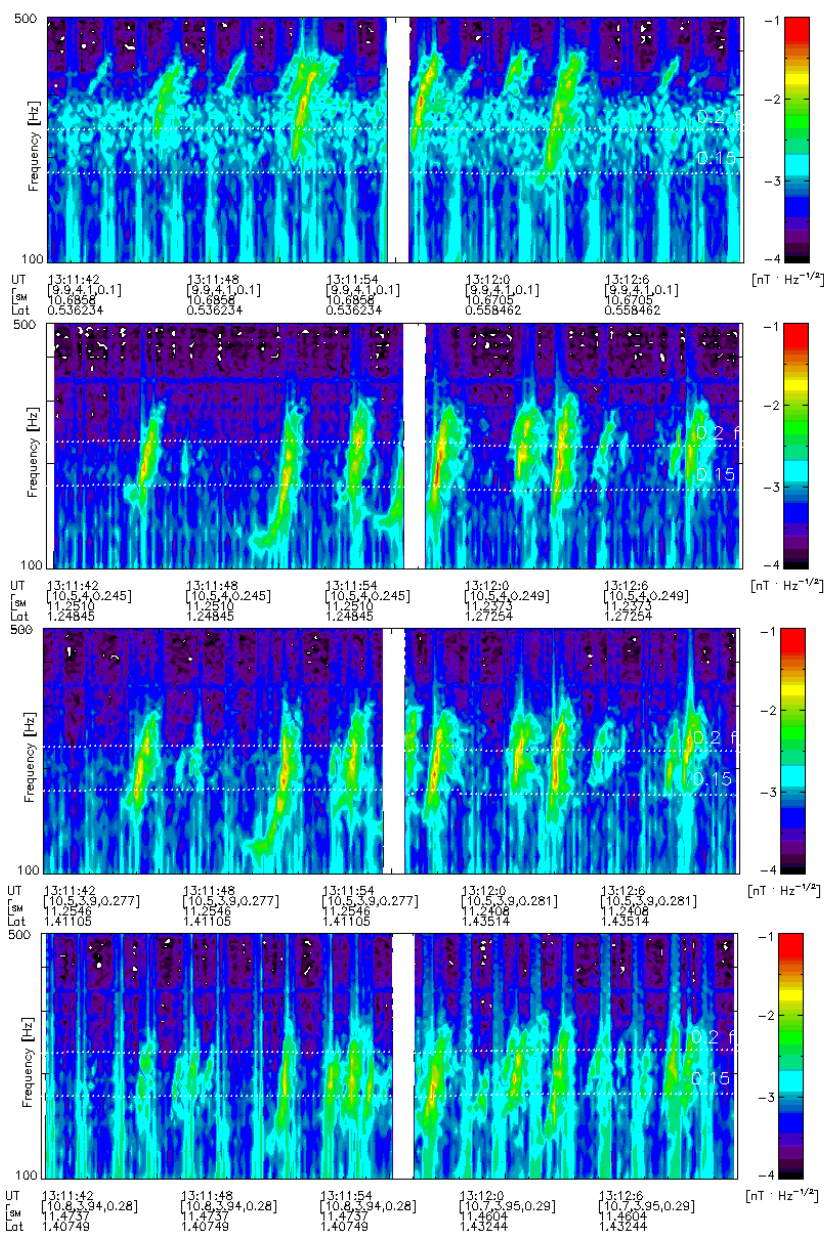

Fig. 1. Detailed time-frequency power spectrograms of magnetic field fluctuations near the source region captured by the SCM instruments onboard four THEMIS spacecraft on 17 July 2007. Panels (from top to bottom) show data from THB, THC, THD, and THE, respectively. Magnetic dipole latitude, magnetic shell, and SM coordinates are given for all s/c. Radial distances are from 10.6 to $11.5 R_{\mathrm{E}}$, and MLT is about 13:30 UT during this interval.

information about background conditions, flux gate magnetometer (FGM) observations (Auster et al., 2008) and plasma measurements of the electrostatic analyzers (ESA) (McFadden et al., 2008) were used. We use spin averaged magnetic field data with a $3 \mathrm{~s}$ resolution and plasma moments with a 3 s resolution.

At about 13:12 UT on 17 July 2007, high-amplitude chorus emissions were captured onboard four THEMIS spacecraft with radial distances from 10.6 to $11.5 R_{\mathrm{E}}$. Electric field measurements were available only for THC, THD, and THE. Detailed time-frequency power spectrograms of magnetic and electric field fluctuations near the source region captured by the SCM instruments onboard the four THEMIS spacecraft THB, THC, THD, and THE are shown in the Figs. 1 and 2, respectively. Their positions are listed in Table 1 .
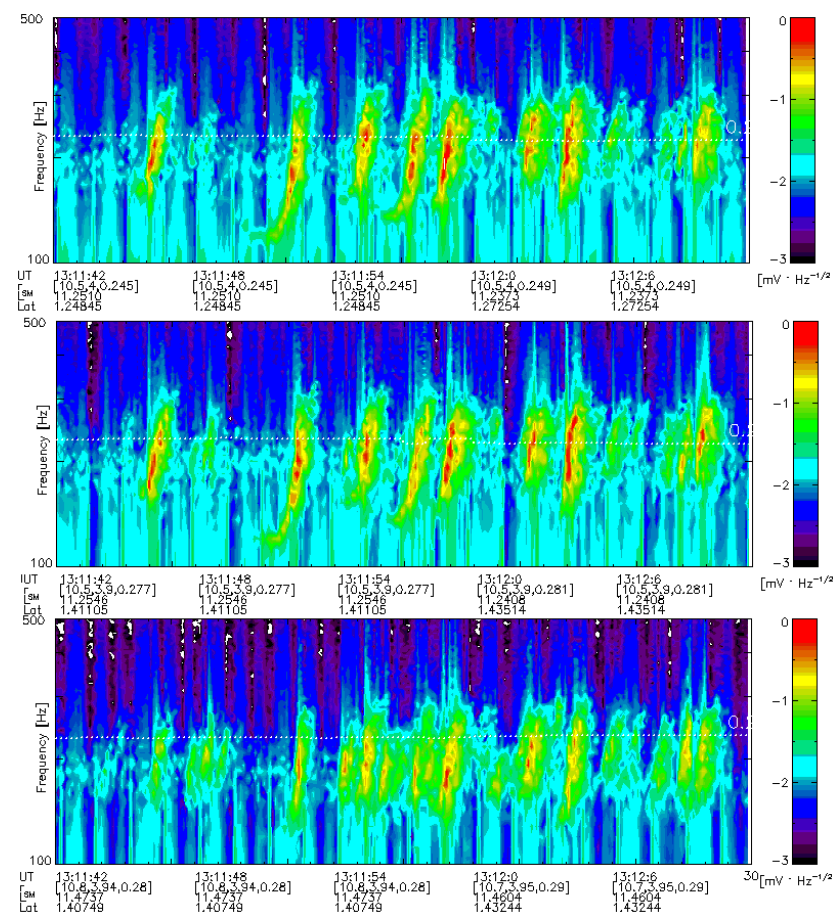

Fig. 2. Detailed time-frequency power spectrograms of electric field fluctuations near the source region captured by the EFI instruments onboard four THEMIS spacecraft on 17 July 2007. Panels (from top to bottom) show data from THC, THD, and THE, respectively. Magnetic dipole latitude, magnetic shell, and SM coordinates are given for all s/c. Radial distances are from 10.6 to $11.5 R_{\mathrm{E}}$, and magnetic local time is about 13:30 UT.

Table 1. Positions of the THEMIS spacecraft and cross spacecraft distances along and transverse to the local magnetic field relative to THC on 17 July 2007 at 13:12:00 UT.

\begin{tabular}{lrlllc}
\hline SC $\mathrm{i}-\mathrm{j}$ & \multicolumn{1}{c}{$X_{\mathrm{SM}}, R_{\mathrm{E}}$} & $Y_{\mathrm{SM}}, R_{\mathrm{E}}$ & $Z_{\mathrm{SM}}, R_{\mathrm{E}}$ & $l_{\|}, \mathrm{km}$ & $l_{\perp}, \mathrm{km}$ \\
\hline THA & 11.8950 & 3.5361 & 0.7120 & 2021.1 & 9754.77 \\
THB & 9.8636 & 4.0663 & 0.1040 & -376.5 & 4215.3 \\
THC & 10.5024 & 3.9734 & 0.2494 & 0.0 & 0.0 \\
THD & 10.5211 & 3.9274 & 0.2813 & 237.5 & 373.3 \\
THE & 10.7474 & 3.9482 & 0.2863 & 251.9 & 1586.0 \\
\hline
\end{tabular}

The wave normal vector $\boldsymbol{k}$ direction is estimated using the singular value decomposition technique (Santolik et al., 2003), which involves computing a spectral matrix consisting of auto-power and cross-power spectra from the three magnetic components. Although this method has an inherent $180^{\circ}$ ambiguity in the wave normal direction, this ambiguity can be removed if the Poynting vector can be determined. Since the wave normal vector must have a component in the direction of the energy flow, a scalar product $(\boldsymbol{S} \times \boldsymbol{k})$ must be positive. 


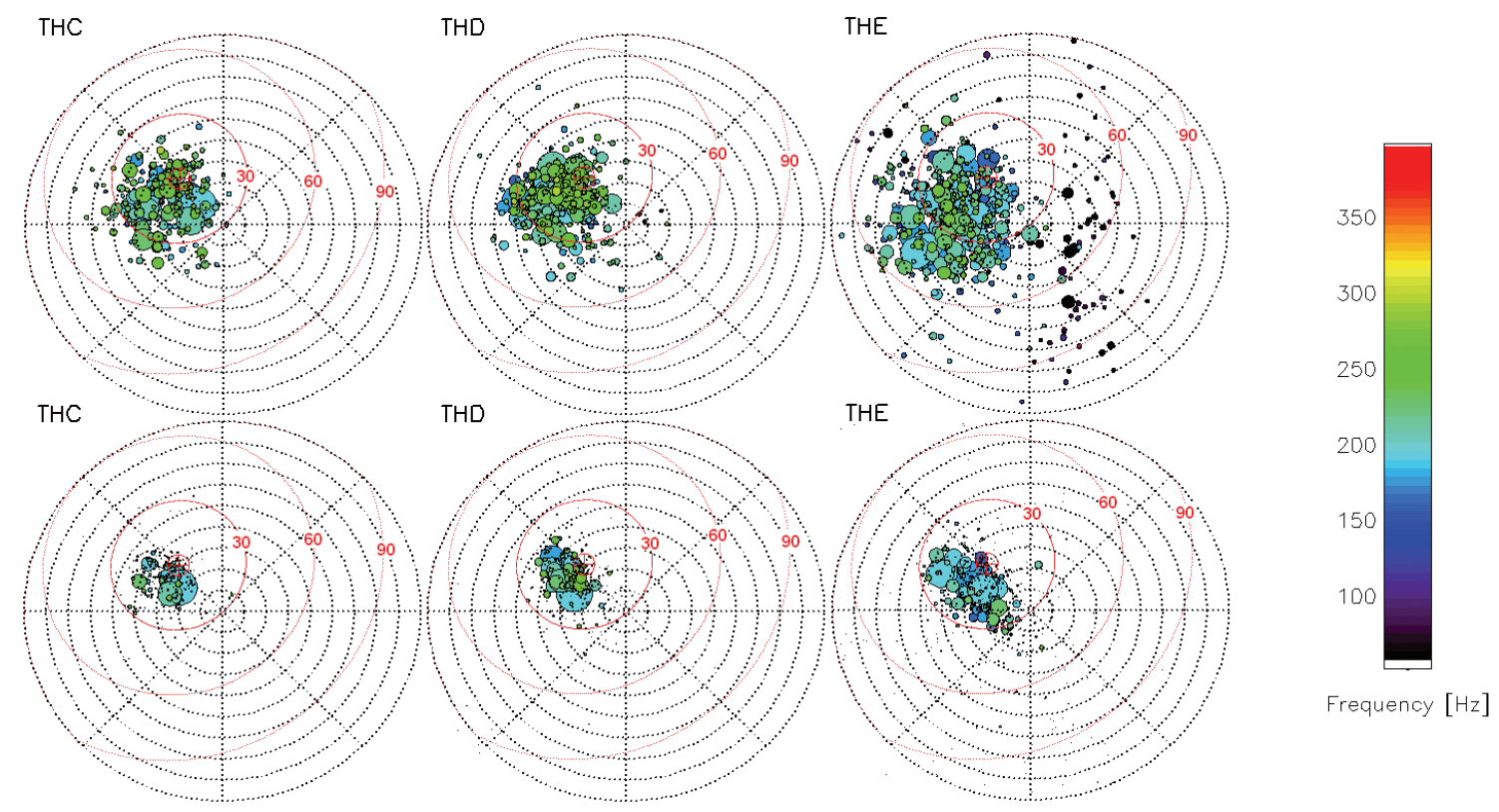

Fig. 3. Dependence of the $\boldsymbol{k}$ directions (top) and Poynting vector directions (bottom) on the frequency in GSE coordinates. Wave amplitude is coded with circle size. The direction of the local magnetic field is shown with a red cross.

The Poynting vector is estimated from the Fourier components as $\boldsymbol{P}=\operatorname{Re}\left(\boldsymbol{E}(\omega) \times \boldsymbol{B}^{*}(\omega)\right)$ (LeDocq, 1998) assuming that $(\boldsymbol{B} \times \boldsymbol{E})=0$. Poynting vector calculations performed with the third component of the electric field (measured by the axial antenna) give the same results.

The direction of $\boldsymbol{k}$ and Poynting flux direction are plotted in Fig. 3. It is clearly seen that whistler waves propagate mainly parallel to the ambient magnetic field. The direction of the Poynting flux indicates that observed whistlers propagate away from the magnetic field minimum of the flux tube.

\section{Correlation analysis}

Chorus waves belong to the whistler wave mode. We shall consider the so-called electron whistler waves in the frequency range $\omega_{\mathrm{LH}}<\omega<\Omega_{\mathrm{e}}$, where $\omega_{\mathrm{LH}}$ is the lower hybrid frequency, and $\Omega_{\mathrm{e}}=|e| B / m_{\mathrm{e}}$ is the electron gyrofrequency. Their refraction index can be found using the dispersion relation in the cold plasma approximation (Helliwell, 1967):

$n^{2}(\omega, \theta)=1+\frac{\omega_{\mathrm{pe}}^{2}}{\omega\left(\Omega_{\mathrm{e}}|\cos \theta|-\omega\right)}$,

where $\omega_{\mathrm{pe}}$ is the electron plasma frequency, and $\theta$ is the angle between the wave $\boldsymbol{k}$ and the background magnetic field. This dependence upon the angle results in anisotropy of the phase and group velocities and dependence of the wave polarization upon the angle. We shall consider multipoint measurements of whistler waves that are supposed to have group velocities almost along the magnetic field. Our goal is to analyze multipoint correlations. In magnetized plasmas anisotropy also results in anisotropic fluctuations that in the simplest case can be described by two characteristic correlation lengths: parallel and perpendicular to the magnetic field. Plasma density inhomogeneities are usually separated into two groups according to their characteristic scale with respect to the wavelength. Small-scale inhomogeneities can scatter whistler mode waves in all directions and profoundly change wave characteristics (Sonwalkar, 2006). Large-scale inhomogeneities with spatial scales comparable to and larger than the wavelength can produce slow variations in wave $\boldsymbol{k}$ amplitude and direction. This can be done using regular procedures based on small perturbations of the unperturbed solution. We describe such an approach making use of the simplified model developed for the wave propagation in random media.

Because we are not interested in the variations of the wave polarization, we use the scalar field mode for simplicity.

The wave-related field perturbation can be written as

$u=U_{0} \exp \{i S+\chi\}$,

where $S$ is the wave phase with regular and random components and $\chi$ is the wave amplitude level. The phase and amplitude levels are denoted as an infinite series following Rytov et al. (1978): $S=S_{0}+S_{1}+S_{2}+\ldots$ and $\chi=\chi_{0}+\chi_{1}+$ $\chi_{2}+\ldots$, where $S_{0}$ and $\chi_{0}$ are solutions for the undisturbed refractive index $\varepsilon=\langle\varepsilon\rangle$. Below we account only for the first term in the series $S_{1}$ and $\chi_{0}$. 
The amplitude level and wave phase fluctuation due to propagation effects are estimated from the Helmholtz equation with some assumptions. Taking advantage of the results of Rytov et al. (1978) and Agapitov et al. (2010), let us consider the wave propagating from the source region in the plasma with large-scale random density fluctuations characterized by two scales $l_{\|}$and $l_{\perp}$ parallel and perpendicular to the background magnetic field. The primary wave is thought to propagate along the magnetic field (Fig. 3). Let us assume that wave propagation can be described in the geometrical optics approximation, which means that $l_{\|}, l_{\perp} \gg \lambda$, $\lambda$ is the wavelength. Without loss of generality we shall consider a monochromatic wave propagation. Under the above conditions the wave phase can be written in the form of the eikonal. With such an approach, one does not consider backward scattering and diffraction effects. Use of the geometric optic approximation is justified if the first Fresnel zone is much smaller than the characteristic inhomogeneity scale of the dielectric permeability (refractive index for wave propagation along the magnetic field):

$\sqrt{\lambda L} \ll l_{\|}, l_{\perp}$.

$L$ is the ray path length - the distance from the generation region to the observation point. The phase and propagation direction fluctuations (but not the amplitude level) can be processed using the geometric optic approximation beyond the bounds of the first Fresnel zone (Rytov et al., 1978). Let us suppose that the refractive index can be separated into mean value and fluctuations:

$\varepsilon(\boldsymbol{r})=\langle\varepsilon(\boldsymbol{r})\rangle+\delta \varepsilon(\boldsymbol{r})$.

Below we use the correlation function of the refractive index described by the anisotropic Gaussian correlation function:

$\Psi_{\varepsilon}(\boldsymbol{r})=\sigma_{\varepsilon}^{2} \exp \left[-\frac{z^{2}}{2 l_{\|}^{2}}-\frac{x^{2}+y^{2}}{2 l_{\perp}^{2}}\right]$,

where the $\mathrm{z}$-axis should be directed along the background magnetic field direction, $l_{\perp}$ perpendicular to it, and the distribution in the perpendicular direction is isotropic. In the framework of assumptions made, the eikonal and amplitude level fluctuations are described by the Helmholtz equation

$\Delta u+k^{2} \varepsilon(\boldsymbol{r}) u=0$.

We are interested in the evaluation of the correlation function of eikonal (and related with the eikonal phase) and of the amplitude level correlation. Both characteristics can be found by using correlation function of the refractive index (Agapitov et al., 2010). Assuming that the primary wave propagates approximately along the background magnetic field $S_{0} \sim k z$, one can find for Gaussian correlations of $\varepsilon$ with two characteristic scales, parallel and perpendicular, the phase correlation function in the following form:

$\Psi_{S}\left(\boldsymbol{r}_{1}, \boldsymbol{r}_{2}\right)=\sqrt{\frac{\pi}{2}} \frac{z_{\min } l_{\|} k^{2} \sigma_{\varepsilon}^{2}}{2\langle\varepsilon(\boldsymbol{r})\rangle} \exp \left[-\frac{\left(\boldsymbol{\rho}_{1}-\boldsymbol{\rho}_{2}\right)^{2}}{2 l_{\perp}^{2}}\right]$.
Here $z_{\min }=\min \left\{z_{1}, z_{2}\right\}, \Psi_{\varepsilon}\left(\boldsymbol{\rho}_{1}-\boldsymbol{\rho}_{2}, \zeta\right)$ - correlation function of the refractive index. It follows then that

$\sigma_{S}^{2}(z)=\sqrt{\frac{\pi}{2}} \frac{z l_{\|} k^{2} \sigma_{\varepsilon}^{2}}{2\langle\varepsilon(\boldsymbol{r})\rangle}$.

From the above, it follows that the phase variance is proportional to the ray path length, the variance of the refractive index, and the effective parallel correlation length.

Another important characteristic is the amplitude level correlation function in the same approximation:

$\Psi_{\chi}(\rho, z)=\frac{z^{3}}{24} \int_{0}^{\infty} \Delta_{\perp}^{2} \Psi_{\varepsilon}(\rho, \xi) d \xi$.

One can find the expression for this correlation function by using the same anisotropic distribution for the refractive index:

$\Psi_{\chi}(\boldsymbol{\rho}, z)=\sqrt{\frac{\pi}{2}} \frac{l_{\|} z^{3}}{l_{\perp}^{4}} \frac{\sigma_{\varepsilon}^{2}}{3\langle\varepsilon\rangle}\left(\frac{\rho^{4}}{8 l_{\perp}^{4}}-\frac{\rho^{2}}{l_{\perp}^{2}}+1\right) \exp \left(-\frac{\rho^{2}}{2 l_{\perp}^{2}}\right)$

The expression for the amplitude variance is obtained

$\sigma_{\chi}^{2}=\frac{\sqrt{2 \pi} z^{3} l_{\|}}{3\langle\varepsilon\rangle l_{\perp}^{4}} \sigma_{\varepsilon}^{2}$.

The amplitude level variance is proportional to the 3 rd degree of the ray path length in the geometric optic approximation. Comparing the amplitude and the eikonal variance, one finds

$\sigma_{\chi}^{2} / \sigma_{S}^{2}=\frac{4 z^{2}}{3 l_{\perp}^{4} k^{2}}$,

which means that the amplitude variance should be substantially smaller than the eikonal variance (the approximation is applicable when $z \lambda \ll l_{\perp}^{2}$ ).

All results are obtained assuming a stationary inhomogeneity distribution. To apply the approximation of stationarity to wave propagation, the following conditions should be satisfied: the characteristic time for wave propagation from source to observer should be small compared to that for density and refractive index variations $\tau$. Tchernov (1977) refers to this condition as quasi-static. On the other hand, the exposure time for registration of the wave packet should be large compared with the characteristic time for variations in the refractive index. For the real data analysis where the homogeneity property can be satisfied only locally, it is widely accepted that one should use the structure function (Tchernov, 1977) determined as

$D_{\varepsilon 12}=\left\langle\left|\varepsilon\left(r_{1}\right)-\varepsilon\left(r_{2}\right)\right|^{2}\right\rangle$.

rather than the correlation function. 

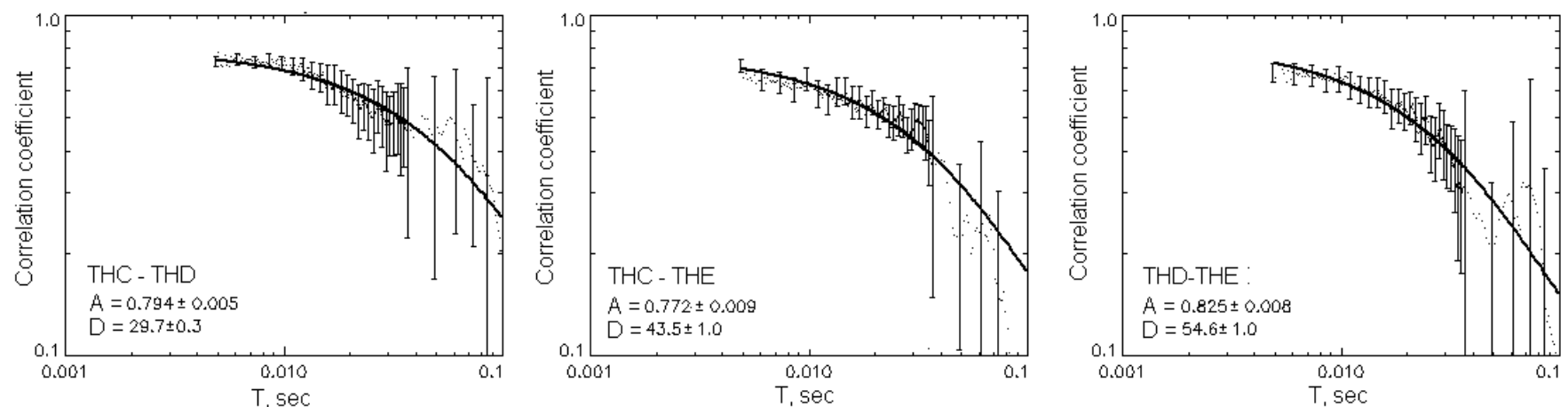

Fig. 4. Cross-correlation analysis of the discrete chorus element observed at 13:12 UT on 17 July 2007 onboard THEMIS spacecraft. The correlation coefficient time dependence is shown for spacecraft THC-THD, THC-THE, and THD-THE, respectively. The approximation with $\frac{\left(1-e^{x}\right)}{x}$ function is shown with solid line. The approximation parameters are listed.

An important property of the correlation function is its asymptotic behavior. With increasing $\boldsymbol{r}$, it approaches zero, and the structure function saturates at some value. Slow spatial variations in the refractive index mean value cannot be distinguished from large-scale fluctuations, which can significantly affect evaluation of the correlation function. Evaluation of the structure function represents the difference between two local points; in this case, large-scale effects are less important. Thus, structure functions can be applied more successfully for analysis when only local homogeneity conditions are satisfied.

Let us consider now the mutual coherence function of the waveform fixed in two points in terms of phase and amplitude level difference:

$\Gamma_{u}\left(\boldsymbol{r}_{1}, \boldsymbol{r}_{2}\right)=\left\langle u\left(\boldsymbol{r}_{1}\right) u^{*}\left(\boldsymbol{r}_{2}\right)\right\rangle$.

In a zone of geometric optic approximation, the phase variance $\sigma_{S}$ can be evaluated more precisely much better than the amplitude variance. Neglecting fluctuations in amplitude with respect to phase, field moment evaluation can be carried out and the field moment can be rewritten as

$u \approx u_{0} e^{i S_{1}}$.

The coherence function can be estimated to be equal to

$\Gamma_{u}\left(\boldsymbol{r}_{1}, \boldsymbol{r}_{2}\right) \approx\left|u_{0}\right|^{2}\left\langle e^{i\left[S_{1}\left(\boldsymbol{r}_{1}\right)-S_{1}\left(\boldsymbol{r}_{2}\right)\right]}\right\rangle$

Making use of the relation $\left\langle e^{i \xi}\right\rangle=e^{-\frac{1}{2}\left\langle\xi^{2}\right\rangle}$ for normally distributed $\xi$ with $\langle\xi\rangle=0$, the following expression is obtained

$\Gamma_{u}\left(\boldsymbol{r}_{1}, \boldsymbol{r}_{2}\right)=I_{0} \exp \left[-\frac{1}{2} D_{\mathrm{S}}\left(\boldsymbol{r}_{1}, \boldsymbol{r}_{2}\right)\right]$,

where $D_{\mathrm{S}}\left(\boldsymbol{r}_{1}, \boldsymbol{r}_{2}\right)=\left\langle\left(\tilde{S}_{1}\left(\boldsymbol{r}_{1}\right)-{\tilde{S_{2}}}_{2}\left(\boldsymbol{r}_{2}\right)\right)^{2}\right\rangle$ is the phase structure function. Taking the exposure time long enough $(t \gg \tau$, $\tau$ is the characteristic time for the plasma media changes) for two signals to be registered at separate points, i. e., having ray paths through the medium where the refractive index can be considered as independent $D_{S}\left(\boldsymbol{r}_{1}, \boldsymbol{r}_{2}\right)=\left(\sigma_{1}^{2}+\sigma_{2}^{2}\right)$. For the $(t \ll \tau) D_{S}\left(\boldsymbol{r}_{1}, \boldsymbol{r}_{2}\right)$ will be a function of $t$ and will grow with the exposure time approximately linearly

$D_{\mathrm{S}}\left(\boldsymbol{r}_{1}, \boldsymbol{r}_{2}\right) \simeq\left(\sigma_{1}^{2}+\sigma_{2}^{2}\right) \frac{t}{\tau}=\sigma_{12}^{2} \frac{t}{\tau}$.

These results inspire us to study the phase characteristics of signals measured in different space locations based on cross-correlation time dependence. Using accumulation procedures can greatly improve the statistics, and the typical dependencies of the accumulated cross correlations can allow us to deduce characteristics of the diffusion coefficients that are dependent upon refractive index fluctuations, as shown above.

It is worth noting that for the two rays propagating along the same path with trajectories that differ only in length, the difference between two diffusion coefficients will be due only to the length of each trajectory. The correlation coefficient of signals at points $\boldsymbol{r}_{1}$ and $\boldsymbol{r}_{1}$ can be found to equal

$K_{u}\left(\boldsymbol{r}_{1}, \boldsymbol{r}_{2}\right)=\frac{1}{\left|u_{0}\right|^{2}} \Gamma_{u}\left(\boldsymbol{r}_{1}, \boldsymbol{r}_{2}\right)$

$=\exp \left[-\frac{1}{2} D_{\mathrm{S}}\left(\boldsymbol{r}_{1}, \boldsymbol{r}_{2}\right)\right]$

The time dependence of the averaged correlation coefficient, similar to that considered before for independent oscillators, can be evaluated as

$$
\begin{aligned}
& \left\langle K_{u}\left(\boldsymbol{r}_{1}, \boldsymbol{r}_{2}\right)\right\rangle_{T}=\frac{1}{T} \int_{0}^{T} K_{u}\left(\boldsymbol{r}_{1}, \boldsymbol{r}_{2}\right) d t \\
& =\frac{1-\exp \left[-\left(\sigma_{1}+\sigma_{2}\right) T\right]}{\left(\sigma_{1}+\sigma_{2}\right) T^{\prime}}
\end{aligned}
$$

A nonlinear least-squares approximation of two free parameters for cross correlation coefficient dependence on estimation time for three THEMIS spacecraft is shown in Fig. 4. 
Function (18) gives minimal chi-square residue compared with power low fit, Gauss fit, or a combination of those fits. Below the average of electric and magnetic field component coefficients is used.

Two ray paths with perpendicular distance between them are taken to be equal to $\rho$ in the transverse direction. The characteristic dependence of the variance should correspond to the single ray path when the rays propagate along the same way, but it should correspond to the sum of two independent diffusion coefficients when the characteristic transverse distance between two ray paths becomes larger than the characteristic perpendicular scale of cross correlations. If the rays propagate close enough to each other that refractive index fluctuations are partially correlated, evaluation of the correlation should take into account the partial correlation.

Taking into account evaluation of the perpendicular characteristic scale, we propose to use the following algorithm to produce an estimate. The transverse dependence of the phase correlation coefficient should have the same Gaussian form as the refractive index:

$K_{\mathrm{S}}\left(z, \rho_{1} ; z, \rho_{2}\right)=e^{-\frac{\left(\rho_{1}-\rho_{2}\right)^{2}}{2 l_{\varepsilon}^{2}}}$.

With partial correlations it is natural to suggest the following dependence of variance:

$\sigma_{12}^{2}=\sigma_{1}^{2}+\sigma_{2}^{2}-2 \sigma_{1} \sigma_{2} K_{S_{1} 2}$.

In addition, $\rho_{1}-\rho_{2}=\rho_{12}, z_{1}-z_{2}=z_{12}$. This system of equations can therefore be represented in the following form:

$\sigma_{12}=\frac{1}{2\langle\varepsilon\rangle} z_{1} l_{\|} \sigma_{\varepsilon}^{2}+\frac{1}{2\langle\varepsilon\rangle} z_{2} l_{\|} \sigma_{\varepsilon}^{2}-2 \frac{1}{2\langle\varepsilon\rangle} \sqrt{z_{1} z_{2}} l_{\|} \sigma_{\varepsilon}^{2} K_{S_{1} 2}$,

To estimate the diffusion coefficient for two separate points with different $\rho$ and $z$, one should divide the trajectory of two ray paths separated in transverse direction till $\left|\rho_{1}-\rho_{2}\right|$ the minimum parallel distance for two propagating rays, let us call it $z_{1}$, and then the part of the trajectory from the point $z_{1}$ to the point $z_{2}$ along the second ray. The entire variance, and consequently the diffusion coefficient, can be presented for each part of the trajectory. The crosscorrelation coefficient $K_{S_{1} 2}$ will be equal to $\exp \left\{-\frac{\left(\rho_{1}-\rho_{2}\right)^{2}}{2 l_{\perp}^{2}}\right\}$ along the part of trajectory where two rays are present. The variance addition along the one single path where only one ray exists can be evaluated in the same way as the variance for auto correlation - by setting the cross correlation coefficient $K_{S_{1} 2}$ equal to zero for this part of the trajectory. This results in the following:

$\sigma_{12}=\frac{1}{2\langle\varepsilon\rangle} z_{1} l_{\|} \sigma_{\varepsilon}^{2}\left[1-\exp \left\{-\frac{\left(\rho_{1}-\rho_{2}\right)^{2}}{2 l_{\perp}^{2}}\right\}\right]+\frac{1}{2\langle\varepsilon\rangle} z_{12} l_{\|} \sigma_{\varepsilon}^{2}$.

Let $z$ be the distance from the source to the closest spacecraft (spacecraft 1).
The procedure employed is based on the solution of the system of equations

$\sigma_{12}=\alpha\left[z\left(1-\exp \left(-\frac{\rho_{12}^{2}}{2 l_{\perp}^{2}}\right)\right)+0.5 z_{12}\right]$.

where the notation $\alpha=\left(\frac{l_{\|} \sigma_{\varepsilon}^{2}}{\langle\varepsilon\rangle}\right)$ is used. The discrete chorus elements shown in Fig. 1 were observed in the frequency range $0.15-0.25$ of the local electron gyrofrequency that is typical for the outer magnetosphere. The field-aligned Poynting flux of whistler emissions shows that they propagate along the magnetic field lines from the magnetic field minimum that is consistent with the waves being generated there. The solution of this system of equations for discrete chorus elements gives the wave generation source position, $l_{\perp}$, and $\left(\frac{l_{\|} \sigma_{\varepsilon}^{2}}{\tilde{\varepsilon}}\right)$. For the obtained case it can be simplified due to independence of the ray path from the source to THE and THD and from the source to THE and THC. The transverse correlation scale can be estimated from relation (24) for spacecraft THC and THD.

The plasma frequency for the selected interval is near $2260 \mathrm{~Hz}$; the gyrofrequency is $1200 \mathrm{~Hz}$. If the wave dispersion relation for whistler waves in a cold plasma is assumed, the wavelength is estimated to be in the range of $220-300 \mathrm{~km}$, and the phase velocity in the range of $(55-60) \times 10^{6} \mathrm{~m} / \mathrm{s}$. A high significance level can be provided due to existence of three magnetic field components and two electric field measured waveforms.

The correlation scale of the refractive index and the electron concentration perturbation are estimated. The phase cross-correlation time dependence gives a correlation scale from 250 to $500 \mathrm{~km}$ transverse to the local magnetic field. The obtained distance to the source region varies from 400 to $2000 \mathrm{~km}$ with source speed about 5-10 thousand $\mathrm{km} / \mathrm{s}$ along the magnetic field line (Fig. 5).

This allows us to conclude that the phase and amplitude level correlation properties can be explained in terms of wave propagation effects caused by random fluctuations of plasma refractive index related to density fluctuations assuming that the wave generation source is coherent in some plane or fluctuates similarly to the density distribution. But can our initial assumptions be justified? Taking the conditions of observations of whistler wave chorus during 17 July 2007 by three THEMIS spacecraft, one finds that although the size of the first Fresnel zone is less than the characteristic inhomogeneity scale, their values are of the same order. The relation $l_{\varepsilon}^{2} \ll l_{\|} \lambda$ is largely satisfied.

Now we evaluate the transverse scale of the chorus waves source region. To quantify the differences between the spectrograms observed near the chorus source region on the four spacecraft, we analyzed spectrogram correlation. The main problem is to distinguish between the source spatial properties and effect of wave propagation through the medium with randomly distributed inhomogeneities affecting the wave's 


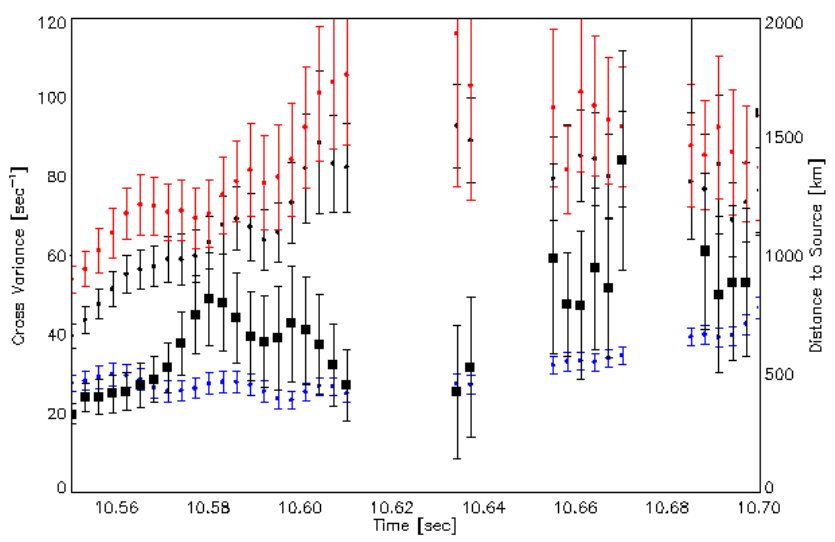

Fig. 5. The time dynamics of variation coefficients $D_{\mathrm{Sij}}$ obtained from waveforms of the THC, THD, THE spacecraft is shown with black, blue and red circles (amplitude is marked on the left scale). The time dynamic of distance from THC spacecraft to the source region is shown with black squares (amplitude is marked on the right scale).

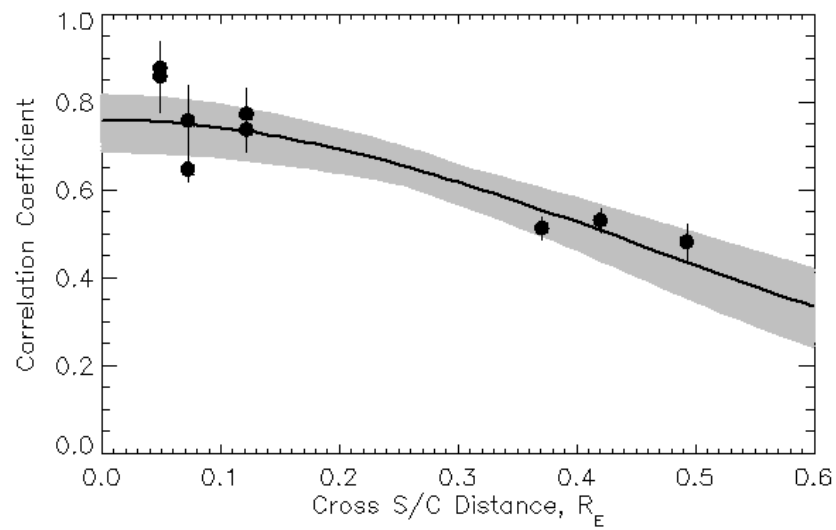

Fig. 6. The amplitude level correlation coefficients of the SCM magnetic field and EFI electric field waveform captured onboard THEMIS spacecraft at 13:12 UT on 17 July 2007 in dependence on cross spacecraft distances.

phase velocity. This can be done if cross distances between observation points vary over a wide range and the source spatial scale is much greater (or much less) than the electron concentration perturbation spatial scale. We used a common time resolution of $0.0625 \mathrm{~s}$, sufficient to distinguish the separate elements and much greater than a random variation time scale. This gives us 480 data points for each spacecraft in a 32-s time interval. We then calculated the cross-correlation coefficients of the common logarithms of these data for each pair of spacecraft. The characteristic scale is estimated by non-linear Gaussian fit of the correlation coefficient dependence on the cross spacecraft distance transverse to the local magnetic field. The generation region size is estimated to be in the range of $2800-3200 \mathrm{~km}$. The dependence of the cor- relation coefficient on the cross-spacecraft distance is shown in Fig. 6. The spatial scale is found to be much greater than the length of whistler wave and also much greater than the refractive index characteristic scale.

\section{Discussion and conclusion}

We present a case study of multispacecraft observations of whistler wave emissions in the outer Earth magnetosphere region near the geomagnetic equator that was not covered in previous work using CLUSTER data. THEMIS project Electric Field Instrument (EFI) and Search Coil Magnetometer (SCM) measurements were used to determine the spatial cor515 relation scale of the chorus source region. We present simultaneous observations of the same chorus elements registered onboard four THEMIS spacecraft in 2007 when all the spacecraft were in the same orbit. The transverse to the background magnetic field cross spacecraft distances varied in the range from 200 to $6000 \mathrm{~km}$.

We propose the novel analysis technique aiming to estimate the characteristics of electron concentration fluctuations by solving the reverse problem of the wave propagation through the random media. This method similar to interferometry technique is based on the multi spacecraft chorus waveform measurements near the source region. It allows one to evaluate the characteristic scales of fluctuations of dielectric constant of the medium along and transverse to the direction of the wave propagation and the characteristic distance to the chorus source.

Since the electron concentration perturbation scale is much smaller than the estimated chorus generation region scale, the analyzed event allows us to obtain a good assumption for the chorus source region scale. Also propagation nearly parallel to the local magnetic field gives an opportunity to distinguish the parallel from the transverse perturbation scale and to estimate the distance to the source along the magnetic field line.

The discrete chorus elements were observed in the frequency range $0.15-0.25$ of the local electron gyrofrequency typical for the outer magnetosphere. The field-aligned Poynting flux of whistler emissions shows that they propagate along the magnetic field lines in the direction away from the magnetic field minimum that is consistent with the waves being generated there. The averaged amplitude correlation analysis allows us to estimate the characteristic spatial halfwidth of the source region transverse to the local magnetic field to be about $2800-3200 \mathrm{~km}$. The correlation scale of refractive index and the electron concentration perturbation are estimated under the geometric optic assumption. The phase cross-correlation time dependence gives a correlation scale from 250 to $500 \mathrm{~km}$ transverse to the local magnetic field. The obtained distance to the source region varies from 400 to $2000 \mathrm{~km}$ with source speed about 5-10 thousands $\mathrm{km} / \mathrm{s}$ along the magnetic field line. 
Acknowledgements. This work was supported by CNES through the grant "Modeles d'ondes", and by the ECO NET program of the EGIDE (France). We acknowledge J. W. Bonnell and F. S. Mozer for use of EFI data, and R. Ergun and Jianbao Tao for help with calibration of the EFI data. We acknowledge K. H. Glassmeier, U. Auster and W. Baumjohann for the use of FGM data provided under the lead of the Technical University of Braunschweig and with financial support through the German Ministry for Economy and Technology and the German Center for Aviation and Space (DLR) under contract 50 OC 0302. THEMIS in the US was supported under NASA contract NAS5-02099. Authors thank to Judy Hohl for very useful assistance in preparation of the manuscript.

Topical Editor I. A. Daglis thanks R. B. Horne for his help in evaluating this paper.

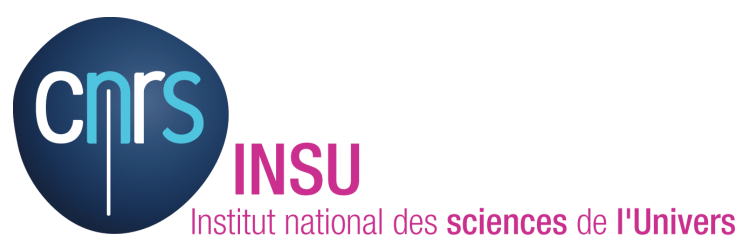

The publication of this article is financed by CNRS-INSU.

\section{References}

Agapitov O.V., V. Krasnoselskikh, T. Dudok de Wit Y. Khotyaintsev O. Santolik, G. Rolland, J. S. Pickett : Multi spacecraft Cluster observations of chorus emissions as a tool for the remote sensing of plasma density fluctuations, J. Geophys. Res., (submited), 2010.

Auster, H., Glassmeier, K. H., Magnes, W., et al.: The THEMIS fluxgate magnetometer, Space Sci. Rev., doi:10.1007/s11214008-9365-9, 2008.

Bonnell, J. W., Mozer, F. S., Delory, G. T., Hull, A. J., Ergun, R. E., Cully, C. M., Angelopoulos, V., and Harvey, P. R.: The Electric Field Instrument (EFI) for THEMIS, Space Sci. Rev., 141, 303341, 2008.

Breneman, A. W., Kletzing, C. A., Pickett, J., Chum, J., and Santolik, O.: Statistics of multispacecraft observations of chorus dispersion and source location, J. Geophys. Res., 114, A06202, doi:10.1029/2008JA013549, 2009.

Burton, R. K. and Holzer, R. E.: The origin and propagation of chorus in the outer magnetosphere, J. Geophys. Res., 79, 10141023, 1974.

Goldstein, B. E. and Tsurutani, B. T.: Wave normal directions of chorus near the equatorial source region, J. Geophys. Res., 89, 2789-2810, 1984.

Gurnett, D. A., Anderson, R. R., Scarf, F. L., Fredricks, R. W., and Smith, E. J.: Initial results from the ISEE 1 and 2 plasma wave investigation, Space Sci. Rev., 23, 103-122, 1979.

Inan, U. S., Platino, M., Bell, T. F., Gurnett, D. A. , and Pickett, J. S.: Cluster measurements of rapidly moving sources of ELF/VLF chorus, J. Geophys. Res., 109, A05214, doi:10.1029/2003JA010289, 2004.

Hattori, K., Hayakawa M., Lagoutte, D., Parrot, M., and Lefeuvre, F.: Further evidence of triggering chorus emissions from wavelets in the hiss band, Planetary and Space Science 39, 11, 1465-1472, 1991.
Hayakawa M., Hattori, K., Lagoutte, D., Parrot, M., and Lefeuvre, F.: Direction finding of chorus emissions in the out magnetosphere and their generation and propagation, Planet. Space Sci., 38, 135-143, 1990.

Helliwell R. A.: Whistlers and related ionospheric phenomena, 1967.

Horne, R. B., Thorne, R. M., Shprits, Y. Y., Meredith, N. P., Glauert, S. A., Smith, A. J., Kanekal, S. G., Baker, D. N., Engebretson, M. J., Posch, J. L., Spasojevic, M., Inan, U. S., Pickett, J. S., and Decreau, P. M. E.: Wave acceleration of electrons in the Van Allen radiation belts, Nature, 437, 227-230, doi:10.1038/nature03939, 2005.

Horne, R. B., Thorne, R. M., Glauert, S. A., Albert, J. M., Meredith, N. P., and Anderson, R. R.: Timescale for radiation belt electron acceleration by whistler mode chorus waves, J. Geophys. Res., 110, A03225, doi:10.1029/2004JA010811, 2005.

LeDocq, M. J., Gurnett, D. A., and Hospodarsky, G. B.: Chorus source locations from VLF Poynting flux measurements with the Polar spacecraft, Geophys. Res. Lett., 25, 4063-4066, 1998.

Le Contel, O., Roux, A., Robert, P., Coillot, C., Bouabdellah, A., de la Porte, B., Alison, The Search Coil Magnetometer for THEMIS, Space Science Reviews, 141, 0038-6308, 2008.

Li, W., Thorne, R. M., Angelopoulos, V., Bonnell, J. W., McFadden, J. P., Carlson, C. W., LeContel, O., Roux, A., Glassmeier, K. H., and Auster, H. U.: Evaluation of whistler-mode chorus intensification on the nightside during an injection event observed on the THEMIS spacecraft, J. Geophys. Res., 114, A00C14, doi:10. 1029/2008JA013554, 2009.

Li, W., Thorne, R. M., Angelopoulos, V., Bortnik, J., Cully, C. M., Ni,B., Le Contel, O., Roux, A., Auster, U., and Magnes, W.: Global Distribution of Whistler-mode Chorus Waves Observed on the THEMIS Spacecraft, J. Geophys. Res., 36, L09104, doi:10.1029/2009GL037595, 2009.

Li, W., Thorne, R. M., Nishimura, Y., Bortnik, J., Angelopoulos, V., McFadden, J. P., Larson, D. E., Bonnell, J. W., LeContel, O., Roux, A., Glassmeier, K. H., and Auster, U.: THEMIS Analysis of Observed Equatorial Electron Distributions Responsible for the Chorus Excitation, J. Geophys. Res., 115, A00F11, doi:10.1029/2009JA014845, 2010.

Meredith, N. P., Horne, R. B., and Anderson, R. R.: Substorm dependence of chorus amplitudes: Implications for the acceleration of electrons to relativistic energies, J. Geophys. Res., 106, 13165-13178, doi:10.1029/2000JA900156, 2001.

Meredith, N. P., Horne, R. B., Thorne, R. M., and Anderson, R. R.: Favored regions for chorus-driven electron acceleration to relativistic energies in the Earth's outer radiation belt, Geophys. Res. Lett., 30(16), 1871, doi:10.1029/2003GL017698, 2003.

McFadden, J. P., Carlson, C. W., Larson, D., Lin, R. P., and Angelopoulos, V.: The THEMIS ESA plasma instrument and in-flight calibration, Space Sci. Rev., 141(1-4), 277-302, doi:10.1007/s11214-008-9440-2, 2008.

Nagano, I., Yagitani, S., Kojima, H., and Matsumoto, H.: Analysis of Wave Normal and Poynting Vectors of the Chorus Emissions Observed by GEOTAIL, J. Geomag. Geoelectr., 48, 299-307, 1996

Omura, Y., Nunn, D., Matsumoto, H., and Rycroft, M. J.: A review of observational, theoretical and numerical studies of VLF triggered emissions J. Atmos. Terr. Phys., 53, 351-368, 1991.

Parrot, M., Santolík, O., Cornilleau-Wehrlin, N., Maksimovic, M., 
and Harvey, C. C.: Source location of chorus emissions observed by Cluster, Ann. Geophys., 21, 473-480, 2003, http://www.ann-geophys.net/21/473/2003/.

Rytov, S. M., Kravtsov, Yu. A., and Tatarskiy, V. I.: Introduction to the statistical radiphysics, Moscow, Science, 2, 463 p., 1978.

Sazhin, S. S. and Hayakawa, M.: Magnetospheric chorus emissions: A review, Planet. Space Sci., 40, 681-697, 1992.

Santolik, O., Parrot, M., and Lefeuvre, F.: Singular value decomposition methods for wave propagation analysis, Radio. Sci., 38(1), 1010, doi:10.1029/2000RS002523, 2003.

Santolik, O. and Gurnett, D.: Transverse dimensions of chorus in the source region, Geophys. Res. Lett., 30(2), 1031, doi:10.1029/2002GL016178, 2003.

Santolik, O., Gurnett, D. A., Pickett, J. S., Parrot, M., and Cornilleau-Wehrlin, N.: Central position of the source region of storm-time chorus, Planet. Space Sci., 53, 299-305, 2005.

Sibeck, D. G. and Angelopoulos, V.: THEMIS science objectives and mission phases, Space Sci. Rev., 141(1-4), 35-59, doi:10.1007/s11214-008-9393-5, 2008.
Sonwalkar, V. S.: The influence of Plasma Density Irregularities on Whistler-Mode Wave Propagation, in: Lect. Notes. Phys. Geospace Electrmagntic Waves and Radiation, edited by: LaBelle, J. W. and Treumann, R. A., Springer, 687, 141-191, 2006.

Tchernov, L. A.: Waves in the randomly inhomogeniuos medium, Moscow, Science, 1977.

Trakhtengerts, V. Y.: A generation mechanism for chorus emission, Ann. Geophys., 17, 95-100, doi:10.1007/s00585-999-0095-4, 1999.

Tsurutani, B. T. and Smith, E. J.: Postmidnight chorus: a substorm Phenomenon, J. Geophys. Res., 79, 118-127, 1974.

Yagitani, S., Nagano, I., Matsumoto, H., Omura, Y., Paterson, W. R., Frank, L. A., and Anderson, R. R.: Wave and particle measurements for chorus emissions by GEOTAIL in the magnetosphere, Adv. Space Res., 24, 91-94, 1999. 